\title{
PROPERTIES PRESERVED UNDER ALGEBRAIC CONSTRUCTIONS
}

\author{
R. C. LYNDON
}

The study of properties of algebras belongs equally to Algebra and to Metamathematics. But here we consider only questions that concern all abstract algebras, and we attempt to discuss them without invoking any unfamiliar metamathematical machinery. In consequence, we can not do justice to the many interesting matters that concern important special classes of algebraic systems, nor to the broad metamathematical context in which the problems mentioned here belong.

Our topic was approached in considerable algebraic generality by Philip Hall and his associates; a pioneering result of Garrett Birkhoff, $1935,{ }^{1}$ is mentioned below. A parallel treatment, from a viewpoint presupposing rather novel metamathematical considerations, has been given by P. Jordan, 1949. On the metamathematical side, our topic can be regarded as a chapter in the "theory of elementary (or arithmetical) classes" expounded by Alfred Tarski in 1950, 1954, and, in independent form, by Leon Henkin, 1953, and Abraham Robinson, 1950; most of the recent work discussed below has been directly influenced by these ideas.

An Example. It is a familiar fact that every homomorphic image of a commutative ring is commutative, while a homomorphic image of a domain need not itself be a domain. The proof, that commutativity is preserved under homomorphism and that the property of being a domain is not, is trivial. This triviality resides, at least in part, in the fact that the proof makes no significant reference to the algebraic meanings of the properties in question, but depends rather only on the form of the axioms expressing these properties. If $F(x, y)$ is any polynomial, the same reasoning shows that every homomorphic image of a ring satisfying the condition

$$
\text { for all } x \text { and } y: F(x, y)=0
$$

will have the same property, while the property expressed by the condition

An address delivered before the Evanston Meeting of the Society on November 28, 1958 by invitation of the Committee to Select Hour Speakers for Western Section Meetings; received by the editors December 28, 1958.

${ }^{1}$ A name accompanied by a date will always indicate a reference to the bibliography. The date is that of the publication cited, and not necessarily the year in which the result quoted was first established. 


$$
\text { for all } x \text { and } y: F(x, y)=0 \Rightarrow x=0 \text { or } y=0
$$

is not, in general, preserved under homomorphism. The relevant distinction is easily seen to lie in the fact that, unlike the commutativity axiom, the domain axiom contains a concealed negation:

$$
\text { for all } x \text { and } y: x y \neq 0 \text { or } x=0 \text { or } y=0 .
$$

Unlike an equation, the negation of an equation need not be preserved under the passage from an algebraic system to a homomorphic image of the system.

This suggests the conjecture that

$\mathrm{H}_{1}$ : every positive sentence is preserved under homomorphism.

Here we consider only "elementary" sentences that can be built up from equations by means of conjunction $(a n d)$, disjunction (or), negation (not), universal quantification (for all $x$ ), and existential quantification (there exists $x$ ); such a sentence is positive if it does not contain negation. The proof of $\mathrm{H}_{1}$ is trivial.

A plausible converse is

$\mathrm{H}_{2}$ : every sentence that is preserved under homomorphism is equivalent to some positive sentence.

Conjecture $\mathrm{H}_{2}$ is also true, but its proof is not trivial. That $\mathrm{H}_{2}$ should be more difficult than $\mathrm{H}_{1}$ is suggested by the fact that, unlike $\mathrm{H}_{1}$, it asserts the existence of something: of a positive sentence equivalent to the given sentence. This suggestion is supported by the fact that

$\mathrm{H}^{*}$ : there is no general method for deciding whether a given sentence is equivalent to some positive sentence.

The result $\mathrm{H}_{2}$ can be strengthened to an "interpolation theorem":

$\mathrm{H}_{2}^{\prime}$ : Suppose that $S$ and $T$ are sentences such that, whenever $S$ holds in an algebraic system $A$, then $T$ holds in every homomorphic image $A^{\prime}$ of $A$. Then there exists a positive sentence $M$ such that $S$ implies $M$, and $M$ implies $T$.

In turn, $\mathrm{H}_{2}^{\prime}$ has a converse:

$\mathrm{H}_{3}$ : Let $S$ be a sentence, and $A^{\prime}$ an algebraic system in which every positive consequence $M$ of $S$ holds. Then $A^{\prime}$ is $(\cdot \cdots)$ a homomorphic image of some system $A$ in which $S$ holds.

As it stands, $\mathrm{H}_{3}$ is not quite true, but it becomes true after a minor technical amendment. Precisely, for "( . . . )" read "an elementary subsystem of"; this concept was introduced by Tarski and R. Vaught, 1957.

The truth of $\mathrm{H}_{1}$ was remarked by Lyndon, 1951, and independently by $\mathrm{E}$. Marczewski, 1951, who conjectured the converse $\mathrm{H}_{2}$. The truth of $\mathrm{H}_{2}$ was asserted by J. Kos in $1955 \mathrm{~b}$, and by A. I. Malcev 
in 1956. Proofs of $\mathrm{H}_{2}^{\prime}, \mathrm{H}_{3}$ and $\mathrm{H}^{*}$ were obtained by Lyndon in 1957a, b and will appear in 1959a, b. Proofs of $\mathrm{H}_{2}^{\prime}$ and $\mathrm{H}_{3}$ by more sophisticated and powerful methods have been obtained by $\mathrm{H}$. J. Keisler, 1958. In an Appendix to this note a sketch of my proof is given.

The general problem. Theorem $\mathrm{H}$, that is, $\mathrm{H}_{1}$ together with its converse $\mathrm{H}_{2}$, exemplifies the following type of theorem:

$\mathrm{T}:$ a sentence is preserved under certain algebraic constructions if and only if it is equivalent to some sentence exhibiting certain formal characteristics.

The formulation of Theorems $\mathrm{T}$ requires some clarification.

As algebraic constructions we have in mind the passage from an algebraic system to a homomorphic image, the passage from a set of systems to their direct product, and so forth, as illustrated in what follows. As formal characteristics of sentences, we have in mind those properties that can be read off by inspection, such as that of not containing the symbol for negation. In each particular theorem of type $\mathrm{T}$, the constructions and formal characteristics under consideration must, of course, be unambiguously defined. But the general question of what sorts of things merit consideration as algebraic constructions or as formal characteristics is perhaps best left as a matter of taste, under which we subsume the criterion of what can be proved about them. It is conceivable, and to an extent true, that a familiar construction may correspond to a characterization that appears reasonable only in retrospect, and conversely.

By an algebra, or algebraic system, we shall always mean what, apart from minor technicalities, can be described as a nonempty set $A$ of "elements," together with a sequence of "operations" $f_{i}$ of specified ranks $n_{i}=0,1,2, \cdots$, that is, of functions from the cartesian product $A^{n_{i}}$ into $A$. Our introductory example, dealing with rings, could be interpreted as dealing with systems with two binary operations, written as addition and multiplication, and a constant (operation of rank 0 ) written as 0 . It was also more or less assumed that we were considering only such systems that were in fact rings, that is, which satisfied the ring axioms. In the example, this assumption does not affect the statement of the theorem. For simplicity, we shall here admit as an algebra any nonempty set equipped with an appropriate sequence of operations, without imposing any special postulates on these operations. Relativization of results to systems subject to certain special axioms ordinarily presents no difficulty, but may alter radically the content of the theorem, as is illustrated by an amusing observation of Abraham Robinson: every sentence in the 
theory of fields that holds for the one-element "field" is preserved under homomorphism; a moment's reflection shows that this jibes with Theorem $\mathrm{H}$, using the appropriate interpretation of "equivalence" of sentences.

Tarski ordinarily considers more general systems that possess relations other than equality, and possibly no operations: for example, ordered groups, ordered sets, geometries defined by incidence or "betweenness" relations. Although the usual devices for translating between functions and relations are not always applicable in the present context, the proofs of the main results apply equally well to these more general systems, and it is only for expository simplicity that we adhere to our narrower definition.

The definition of sentence given above will serve, provided, to account for pathological cases, we admit to our notation two onesymbol sentences, the one identically false and the other true. It remains, by way of elucidating the content of Theorems $T$, to define the relation, of a sentence to a system, that is expressed by saying that the sentence holds or is true in the system, and the relation between sentences that says they are equivalent. Although a precise algebraic definition of when a sentence holds in a system, as given for example in Tarski, 1950, is somewhat involved in detail, it does not depart from common intuition, which for the restricted sort of sentences considered here is quite reliable. Two sentences may then be defined to be equivalent if they hold in exactly the same systems. It may be noted that the relation of one sentence implying another can be given a similarly semantic or "behavioristic" definition, and in this spirit we can free ourselves entirely of the theory of formal deduction in mathematical logic.

It is, of course, far from true that all algebraically interesting properties are expressible by sentences of the limited sort under consideration. The discussion of properties expressible by an infinite set of such sentences taken "in conjunction" (for example, characteristic zero), or "in disjunction" (finite characteristic), is possible in the full development of the general theory, as in Henkin, Robinson, and Tarski. A more radical step has been taken by Jordan in considering "infinitely long sentences" (for example, the property of being a torsion group); such sentences have been studied by C. Karp, 1958.

But, from the point of view of classical algebra, the most serious limitation in our definition of sentence lies in the exclusion of variables ranging over subsets of systems, such as ideals or subgroups; we are powerless, with such sentences, to express the ascending chain conditions. The difficulties here lead into the most delicate questions 
of set theory. However, a substantial relaxation of this limitation is promised by recent work of Tarski, 1958, extending results to sentences with variables interpreted as ranging over finite sequences of elements from an algebraic system. With such means one can speak about variable polynomials, or variable ideals in a Nötherian ring. A discussion of the Lefschetz principle of algebraic geometry by A. Seidenberg, 1958, although it does not take into account these latest results, is suggestive of their scope.

There is one other recent development that I regard as an important step away from "sentences" viewed as syntactical entities consisting of a string of symbols in some more or less restricted formal logic, and in the direction of the semantic "properties" of algebraic systems that they express. Keisler has established a number of results in a context where equations have been displaced from their distinctive role as the atomic building blocks in constructing sentences, in favor of an almost arbitrary set of formulas. This has the conceptual advantage of eliminating many irrelevancies resulting from the choice of a particular set of operations or relations in axiomatizing a class of algebraic systems, and the practical advantage of simplifying the proofs of many theorems.

Returning to theorems of type $\mathrm{T}$, we note here that for the most part analogs of the variants of Theorem $\mathrm{H}$ hold; but, for simplicity's sake, we refrain from discussing them. We remark also that, as with $\mathrm{H}$, the "if" of Theorems T seems always to be easy, while the converse may present considerable difficulty.

Some results. Theorem $\mathrm{H}_{1}$ has an analog that is, if anything, even more intuitively obvious:

$\mathrm{E}_{1}$ : every existential sentence is preserved under extension of a system to a containing system.

Here we are considering prenex sentences, consisting of a succession of quantifiers followed by a propositional part that contains no quantifiers; every sentence is equivalent to one of this form. A prenex sentence is then called existential if all its quantifiers are existential quantifiers, and universal if they are all universal. This theorem and its converse were obtained by Henkin, 1956, and "relativized" by Robinson, 1956. They may be regarded as the dual, and a consequence, of a result of Tarski, 1954, and Łoś, 1955a:

$\mathrm{U}:$ a sentence is preserved under passage from systems to subsystems if and only if it is equivalent to some universal sentence.

It may be noted here that Theorem $\mathrm{H}$ likewise has an obvious dual.

Theorems $\mathrm{U}$ and $\mathrm{H}$ can be combined in a sense: 
$\mathrm{UH}:$ a sentence is preserved both under passage to subsystems and under homomorphisms if and only if it is equivalent to some universal positive sentence.

This is not quite an immediate corollary of $\mathrm{U}$ and $\mathrm{H}$ taken together, but its proof involves less than their proofs.

The earliest theorem of the type under consideration, proved by G. Birkhoff in 1935, is a triple combination of this sort:

$\mathrm{UHD}$ : a sentence is preserved under passage to subsystems, to homomorphic images, and to direct products, if and only if it is equivalent to a universally quantified conjunction of equations.

A universally quantified conjunction of equations is of course equivalent to a conjunction of universally quantified equations, or identical equations. Birkhoff in fact proved the stronger result (comparable to $\mathrm{H}_{2}^{\prime}$ with $\mathrm{H}_{3}$ ) that a set of algebraic systems is closed under the three constructions mentioned if and only if it consists of all systems that satisfy some set, possibly infinite, of identical equations. Although Theorem UHD can be obtained easily from UH, Birkhoff's proof of the stronger result, using free algebras, is simpler and more purely algebraic in spirit.

Given Theorems U, H, UH and UHD, symmetry demands also $\mathrm{UD}, \mathrm{HD}$ and D. First a remark about direct products. A theorem of R. Vaught, 1954, that every sentence preserved under finite direct products is also preserved under infinite direct products, clearly makes it necessary to consider only direct products of no more than two factors. Obviously every property is preserved under direct products with a single factor. To simplify the discussion, we choose to admit among direct products the trivial, one-element, direct products of zero factors, and all that we say will be tacitly adapted to this minor departure from common usage.

A class of sentences, now called Horn sentences, was discussed by Alfred Horn in 1951. These are prenex sentences whose propositional part is a conjunction of clauses of the form

$$
e_{1} \text { and } e_{2} \text { and } \cdots \text { and } e_{n} \Rightarrow e_{0} \text {, }
$$

$n \geqq 0$, where the $e_{i}$ are equations. Developing an idea of J. C. C. McKinsey, 1943, Horn established essentially the following result:

UD: a universal sentence is preserved under direct products if and only if it is equivalent to some universal Horn sentence.

This is illustrated by the fact that the domain axiom

$$
\text { for all } x \text { and } y: x y=0 \Rightarrow x=0 \text { or } y=0
$$

is not a Horn sentence, while its consequence (assuming associativity but not commutativity) 


$$
\text { for all } x: x^{2}=0 \Rightarrow x=0,
$$

is a Horn sentence and is preserved under direct product.

The proof of the obvious formulation of a Theorem HD presents no difficulty. That of a Theorem $\mathrm{D}$ does, and we shall return to this matter.

Horn showed that what we call Horn sentences constitute the largest class of prenex sentences, characterized by reference to the structure of their propositional part alone, that are preserved under direct product. All the characterizations considered thus far apply to prenex sentences, and consist of separate conditions on the arrangement of quantifiers and upon the propositional part. Another theorem of the same nature is that of Łoś and R. Suszko, 1955 and of C. C. Chang, 1956:

A: a sentence is preserved under unions of ascending chains if and only if it is equivalent to a universal-existential sentence:

Here a universal-existential sentence consists first of universal quantifiers, next of existential quantifiers, and then a propositional part. In the same vein Keisler, 1958, describes a somewhat more complicated algebraic construction corresponding to prenex sentences with a prescribed number of alternations between universal and existential quantifiers.

A simpler theorem of Keisler, 1958, uses a characteristic that does not "separate" in the manner mentioned above:

$\mathrm{L}:$ a sentence is preserved under passage to direct limits if and only if it is equivalent to some universal-existential sentence in which each existentially quantified variable occurs only positively (that is, not within the scope of a negation).

Another result of the same mixed nature concerns subdirect products. Let a formula be called positive if it can be built up from equations by means of conjunction, disjunction, and quantification alone. Define a special Horn sentence to be any sentence, not necessarily prenex, that is a universally quantified conjunction of clauses of the form $P \Rightarrow e$ where $P$ is a positive formula and $e$ an equation. I established in $1957 \mathrm{c}, \mathrm{d}, 1959 \mathrm{c}$, that:

$\mathrm{S}: A$ sentence is preserved under formation of subdirect products if and only if it is equivalent to some special Horn sentence.

An example of a special Horn sentence is provided by the condition that an associative ring have trivial Jacobson radical:

for all $z$ : [ for all $x$ and $y$ there exists $u$ such that: $x z y+u=x z y u$ and

$$
x z y u=u x z y] \Rightarrow z=0 \text {. }
$$


The consequence ( $\left.1^{\prime}\right)$ of the "domain axiom" (1), cited above, is a special Horn sentence, and, in fact, every associative ring satisfying $\left(1^{\prime}\right)$ is isomorphic to a subdirect product of rings satisfying (1); this is comparable to $\mathrm{H}_{2}^{\prime}$ together with $\mathrm{H}_{3}$, and to Birkhoff's theorem. In this connection it is perhaps curious that I can find no sentence that asserts, of a semisimple ring, without assuming either commutativity or a finiteness condition, that it is simple.

Every special Horn sentence is clearly equivalent to a Horn sentence. The following is a familiar example of a Horn sentence that is not equivalent to any special Horn sentence:

\section{there exists $x$ such that, for all $y: x y=y$.}

Problems. That all is not easy is shown by the lack of a Theorem $\mathrm{D}$, characterizing those sentences that are preserved under formation of direct products. ${ }^{2}$ Since it may be, as Chang and Morel, 1958, suggest, that no reasonable such characterization exists, it is perhaps better to speak of Problem D, of the existence of such a characterization. This is still slightly vague, but takes a precise form in various special contexts.

Theorems UD and HD indicate that every universal or positive sentence preserved under direct product is equivalent to a Horn sentence. I have established (1958b) the same for existential sentences. Further related results are given by $\mathrm{K}$. Bing, 1955. It seems probable that every universal-existential sentence preserved under direct products is equivalent to a Horn sentence; but an example by Chang and Anne Morel, of which we shall speak later, shows that the analogous statement for existential-universal sentences fails.

One further positive result concerns what would seem to be the most trivial class of algebras, in which there are no operations. Sentences about such algebras must be built solely from equations between variables, and can not do more than express certain conditions on the order, or cardinality, $N$, of a system. The property of not having order $p$, where $p$ is a prime, is clearly preserved under direct products, and Chang devised the following Horn sentence, which expresses that $N \neq 3$ :

$$
\begin{gathered}
\text { for all } x_{1}, x_{2}, x_{3} \text { there exists } y \text { such that: } \\
y=x_{1} \Rightarrow x_{2}=x_{3}, \text { and } y=x_{2} \Rightarrow x_{3}=x_{1}, \\
\text { and } y=x_{3} \Rightarrow x_{1}=x_{2} .
\end{gathered}
$$

${ }^{2}$ Added in proof. The important work of A. Oberschelp, 1958, on this question has just come to our attention. 
K. I. Appel, 1958, by an ingenious combinatorial argument, has shown that all sentences in this theory that are preserved under direct product are equivalent to Horn sentences; the best sentence expressing that $N \neq 5$ obtainable by uniform methods contains $5^{50}$ variables.

The counterexample of Chang and Morel, 1956, 1958, is a sentence expressing that a system is a Boolean algebra which (unless it is trivial) contains an atom. It is easy to see that this property is preserved under direct products. To show that the property is not expressible by any Horn sentence, they used a construction, as did Łoś in a different connection, that falls within what are now called reduced products. Following T. E. Frayne, Dana Scott, and Tarski, 1958 (see also S. Kochen, 1958), except in notation, let $A^{I}$ denote the ordinary direct product of algebras $A^{i}$, where $i$ runs over an index set $I$. If $J$ is an ideal in the Boolean algebra of all subsets of the set $I$, let the reduced product $A^{I} / J$ be the result of identifying in $A^{I}$ any two elements whose components differ only on a set of indices that belongs to $J$.

Chang and Morel showed, essentially, that

$\mathrm{R}_{1}$ : every Horn sentence is preserved under reduced products.

That the property of possessing an atom is not expressible by a Horn sentence, they now show by taking $I$ infinite, each $A^{i}$ a two element algebra, and $J$ the set of all finite subsets of $I$.

Chang has raised the question of whether the converse $R_{2}$ of $R_{1}$ holds. I have, departing slightly from the present definition of algebra, considered the seemingly rather trivial class of sentences that do not contain equality nor any operations, and only symbols for relations (or predicates) $r_{i}(x)$ of a single argument. I have shown $(1958 \mathrm{~b})$ that if such a sentence is preserved under direct products, then it is preserved under reduced products. Thus a counterexample analogous to that of Chang and Morel is ruled out. But, in the absence of a proof of $R_{2}$, it is not known whether such a sentence is equivalent to a Horn sentence.

We conclude with a result of Łoś, 1955b, which has been reworked and improved by Frayne, Scott and Tarski, 1958 (also Morel, Scott, Tarski, 1958):

P: all sentences are preserved under formation of reduced products in which the ideal $J$ is a prime ideal.

Appendix. We give here, as an illustration of methods in this theory, an informal sketch of the ideas in our proof (1957a, b, 1959a, b) of Theorem $\mathrm{H}_{2}^{\prime}$, which tend to be obscured in a thoroughly rigorous exposition. 
It is convenient to regard the relation of "equality" between elements of an algebra simply as a relation that satisfies the usual axioms $C(=)$, expressing reflexivity, symmetry, transitivity, and substitutivity, rather than as "identity" in any absolute sense. (This viewpoint is analyzed in G. Zubieta, 1957.) By a familiar device, any homomorphic image of an algebra $A_{1}$, with equality $={ }_{1}$, may be regarded as an algebra $A_{2}$ with the same elements and operations as $A_{1}$, but with a new equality $=_{2}$, which satisfies the condition

$$
I\left(=_{1},=_{2}\right): \text { for all } x \text { and } y: x={ }_{1} y \Rightarrow x={ }_{2} y .
$$

Conversely, from a set $A$ with operations, and with relations $=_{1}$ and $={ }_{2}$ that satisfy $C\left(=_{1}\right), C\left(=_{2}\right)$ and $I\left(={ }_{1},=_{2}\right)$, we can recover $A_{1}$ and $A_{2}$ such that $A_{2}$ is a homomorphic image of $A_{1}$.

In view of this, the hypothesis of Theorem $\mathrm{H}_{2}^{\prime}$, that when $S$ holds in any system $A_{1}$, then $T$ holds in every homomorphic image $A_{2}$, can be rephrased as follows:

$$
S\left(={ }_{1}\right), C\left(={ }_{1}\right), C\left(={ }_{2}\right), I\left(={ }_{1},=_{2}\right) \Rightarrow T\left(={ }_{2}\right) .
$$

The "implication" $\Rightarrow$ in (1) may be taken in a purely formal sense, all the relevant, and usually tacit, reservations regarding admissible interpretations of $=_{1}$ and $=_{2}$ having been incorporated explicitly into the hypotheses $C\left(=_{1}\right), C\left(=_{2}\right)$, and $I\left(=_{1},=_{2}\right)$. It will be useful to rewrite (1) in the equivalent form

$$
S\left(={ }_{1}\right) \text { and } C\left(=_{1}\right) \text { and } I\left(=_{1},=_{2}\right) \Rightarrow\left[C\left(={ }_{2}\right) \Rightarrow T\left(=_{2}\right)\right] \text {. }
$$

We digress momentarily to illustrate an "interpolation theorem" of W. Craig, 1958a, b, in the domain of mathematical logic. Let $r_{1}, r_{2}$ and $s$ be relations, and $U\left(r_{1}, s\right)$ a sentence that contains $r_{1}$ and $s$ but not $r_{2}$, while $V\left(r_{2}, s\right)$ is a sentence containing $r_{2}$ and $s$ but not $r_{1}$. Suppose that $U\left(r_{1}, s\right)$ implies $V\left(r_{2}, s\right)$. Then Craig's theorem implies the existence of a sentence $M(s)$, containing $s$ but neither $r_{1}$ nor $r_{2}$, such that

$$
U\left(r_{1}, s\right) \Rightarrow M(s) \Rightarrow V\left(r_{2}, s\right) .
$$

Evidently, in some sense, in passing from " $U$ implies $V$ " to " $M$ implies $V$, we have obtained $M$ from $U$ by eliminating hypotheses that are irrelevant to the proof of $V$. To refine Craig's theorem, we may suppose all sentences written in such a way that the negation symbol never occurs except immediately preceding one of $r_{1}, r_{2}$ or $s$. Replace $U$, as above, now by $U\left(r_{1}, r_{2}^{+}, s\right)$, where $U$ may now contain $r_{2}$, but only positively, that is, not preceded by the negation symbol. Let $V\left(r_{2}, s\right)$ be as before. Then, if $U$ implies $V$, there exists $M\left(r_{2}^{+}, s\right)$ that 
now may contain $r_{2}^{+}$, but only positively, such that

$$
U\left(r_{1}, r_{2}^{+}, s\right) \Rightarrow M\left(r_{2}^{+}, s\right) \Rightarrow V\left(r_{2}, s\right) .
$$

The same holds if the relation $s$ is entirely missing:

$$
\text { if } U\left(r_{1}, r_{2}^{+}\right) \Rightarrow V\left(r_{2}\right) \text {, then } U\left(r_{1}, r_{2}^{+}\right) \Rightarrow M\left(r_{2}^{+}\right) \Rightarrow V\left(r_{2}\right) \text {. }
$$

We may apply this last to the situation (2), for the expression on the left contains $=_{2}$ only once, in $I\left(=_{1},=_{2}\right) \equiv$ : all $x, y: x \neq \neq_{1} y$ or or $x={ }_{2} y$, and this occurrence is positive, while the expression on the right does not contain $=_{1}$ at all. It follows that there exists $M\left(=_{2}\right)$, containing only $=_{2}$, and this only positively, such that

$$
S\left(={ }_{1}\right) \text { and } C\left(=_{1}\right) \text { and } I\left(={ }_{1},=_{2}\right) \Rightarrow M\left(=_{2}\right)
$$

and that

$$
M\left(=_{2}\right) \Rightarrow\left[C\left(=_{2}\right) \Rightarrow T\left(=_{2}\right)\right] .
$$

If, in (3) and (4), we replace both $=_{1}$ and $=_{2}$ by $=$, we obtain

$$
\begin{gathered}
S(=) \text { and } C(=) \Rightarrow M(=), \\
M(=) \text { and } C(=) \Rightarrow T(=),
\end{gathered}
$$

which indeed say that, in any system with a relation $=$ satisfying the usual axioms for equality, $S$ implies $M$ and $M$ implies $T$.

Our refinement of Craig's theorem remains to be proved, and concerning this proof we make only one or two remarks. First, the idea of eliminating superfluous hypotheses can be used, with a little care, to prove the analogous result for formulas that contain no quantifiers. Second, the general result follows from this, but appears to require G. Gentzen's proof-theoretic calculus, or some equally complicated and not universally familiar machinery. In 1958 and 1959a we have replaced the Gentzen calculus by an algebraic parallel, following ideas of E. Beth, 1953, and others; although this seems appropriate, and is useful for other purposes, it can not be claimed to simplify the overall proof. The new proof of Keisler, 1958, seems altogether preferable to ours in that it entirely dispenses with the Gentzen argument, or any close analog. It appears to transfer an induction, originally implicit in the arguments borrowed from logic, to the algebraic level; here, although requiring some novel algebraic constructions, the argument seems to proceed much more simply.

\section{Bibliography}

K. I. Appel, 1958. Horn sentences in identity theory, Notices Amer. Math. Soc. vol. 6, p. 343. 
E. W. Beth, 1953. On Padoa's method in the theory of definition, Indag. Math. vol. 15 , p. 66.

K. Bing, 1955. On arithmetical classes not closed under direct union, Proc. Amer. Math. Soc. vol. 6, p. 836.

G. Birkhoff, 1935. On the structure of abstract algebras, Proc. Cambridge Philos. Soc. vol. 31, p. 433.

C. C. Chang, 1956. On the unions of chains of models, Bull. Amer. Math. Soc. vol. 62, p. 598.

p. 120.

C. C. Chang and A. C. Davis (Morel), 1956. Arithmetical classes closed under direct product, Bull. Amer. Math. Soc. vol. 62, p. 411.

C. C. Chang and A. C. Morel, 1958. On closure under direct product, J. Symb. Logic vol. 23, p. 149.

W. Craig, 1957a. Linear reasoning. A new form of the Herbrand-Gentzen theorem, J. Symb. Logic vol. 22, p. 250.

,$- 1957 \mathrm{~b}$. Three uses of the Herbrand-Gentzen theorem in relating model theory and proof theory, J. Symb. Logic vol. 22, p. 269.

T. E. Frayne, D. S. Scott and A. Tarski, 1958. Reduced products, Notices Amer. Math. Soc. vol. 5, p. 673.

L. Henkin, 1953. Some interconnections between modern algebra and mathematical logic, Trans. Amer. Math. Soc. vol. 74, p. 410.

- 1956. Two concepts from the theory of models, J. Symb. Logic vol. 21, p. 28.

A. Horn, 1951. On sentences which are true of direct unions of algebras, J. Symb. Logic vol. 16, p. 14.

P. Jordan, 1949. Zur Axiomatik der Verknüpfungsbereiche, Abh. Math. Sem. Univ. Hamburg vol. 16, p. 54 .

C. R. Karp, 1958. Formalizations of functional languages with wffs of infinite length, Notices Amer. Math. Soc. vol. 5, p. 172.

H. J. Keisler, 1958. The theory of models with generalized atomic formulas, I and II, Notices Amer. Math. Soc. vol. 5, pp. 676, 621.

S. Kochen, 1958. Filtration systems, I and II, Notices Amer. Math. Soc. vol. 5, pp. 67,671 .

J. Łos, 1955a. On the extending of models, I, Fund. Math. vol. 42, p. 38.

, 1955b. Quelques remarques, théorèmes et problèmes sur les classes définissables d'algèbres, Studies in Logic and Foundations of Mathematics, North Holland, Amsterdam, p. 98.

J. Łos and R. Suszko, 1955. On the infinite sum of models, Bull. Acad. Polon. Sci. Cl. III vol. 3, p. 201.

R. C. Lyndon, 1951. Review of Horn, 1951, J. Symb. Logic vol. 16, p. 216.

- 1957a. On positive formulas in the predicate logic, Bull. Amer. Math. Soc. vol. 63, p. 282.

, 1957b. On positive classes of relational systems and algebras, Bull. Amer. Math. Soc. vol. 63, p. 282.

, 1957c. On special Horn sentences, Bull. Amer. Math. Soc. vol. 63, p. 356.

, 1957d. On subdirect products of relational systems, Bull. Amer. Math. Soc. vol. 63 , p. 356 .

-1958a. A semantic Herbrand-Gentzen lemma, Notices Amer. Math. Soc. vol. 5, p. 464.

1958b. Direct products of algebras, Notices Amer. Math. Soc. vol. 5, p. 848. 
vol. 9 , p. 129.

1959a. An interpolation theorem in the predicate calculus, Pacific J. Math. 143

, 1959b. Properties preserved under homomorphism, Pacific J. Math. vol. 9,

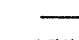
1959c. Properties preserved in subdirect products, Pacific J. Math. vol. 9, p. 155.

, Existential Horn sentences, to appear in Proc. Amer. Math. Soc.

A. I. Malcev, 1956. Subdirect products of models, Dokl. Akad. Nauk SSSR vol. 109, p. 264.

E. Marczewski, 1951. Sur les congruences et les propriêtés positives d'algèbres abstraites, Colloq. Math. vol. 2, p. 220.

J. C. C. McKinsey, 1943. The decision problem for some classes of sentences without quantifiers, J. Symb. Logic vol. 8, p. 61.

A. C. Morel, D. S. Scott and A. Tarski, 1958. Reduced products and the compactness theorem, Notices Amer. Math. Soc. vol. 5, p. 674.

A. Oberschelp, 1958. Über die Axiome produkt-abgeschlossener arithmetischer Klassen, Archiv für Math. Logik und Grundlagenforschung vol. 4, p. 95.

A. Robinson, 1950. On the metamathematics of algebra, Studies in Logic and Foundations of Mathematics, North Holland, Amsterdam.

- 1956. Note on a problem of Henkin, J. Symb. Logic vol. 21, p. 33

A. Seidenberg, 1958. Comments on Lefschetz's Principle, Amer. Math. Monthly vol. 65, p. 685 .

A. Tarski, 1950. Some notions and methods on the borderline of algebra and metamathematics, Proceedings of the International Congress of Mathematicians, Cambridge, vol. 1, p. 705.

, 1954. Contributions to the theory of models, I and II, Indag. Math. vol. 16, pp. $572,582$.

- 1958. Some model-theoretic results concerning weak second-order logic, Notices Amer. Math. Soc. vol. 5, p. 673.

A. Tarski and R. L. Vaught, 1957. Arithmetical extensions of relational systems, Compositio Math. vol. 13, p. 81.

R. L. Vaught, 1954. On sentences holding in direct products of relational systems, Proceedings of the International Congress of Mathematicians, Amsterdam, vol. 2, p. 409.

R. G. Zubieta, 1957. Clases matematicas definadas sin igualdad, Bol. Soc. Mat. Mex. vol. 2, p. 45.

UNIVERSITY OF MiCHIGAN 